\title{
CERAMIC DISKS AS EFFICIENT AND ROBUST CATHODES
}

\author{
I. Boscolo \\ University and INFN, Dipartimento di Fisica via Celoria 16, 20133 Milano, Italy
}

\begin{abstract}
Electron emission from ceramic disks depends strongly on the shape of the front electrode. The common interconnected grid is not good for a stable emission. An electrode consisting of an ensemble of metal point-like islands within a metal ring leads to quite a stable emission. In the first case the sandwich of the continuous electrode (on one surface) and the grating (on the front surface) constrains the domain and the charge carriers to move within the zones covered by the stripes, whilst the quasi-open patchy surface allows the domain switching and in turn the electron flux over the whole area.
\end{abstract}

\section{INTRODUCTION}

The emission presented in the paper refers to energetic electrons expelled in a ceramic disk when excited by a fast high voltage pulse applied to the electrodes deposited on the two surfaces $[1,2]$. The so-called relaxors and antiferroelectric ceramics with fast transition under the action of an electric field are suitable for electron emission [3]. Lead lanthanum zirconate titanate (PLZT) was used in the emission experiments, in proportions of 4/95/5 and $8 / 65 / 35$, where the numbers refer respectively to the lanthanum, zirconium, and titanium atomic percentage. The two ceramics are in the antiferroelectric and ferroelectric (AFE-Fe) phase respectively at room temperature [3].
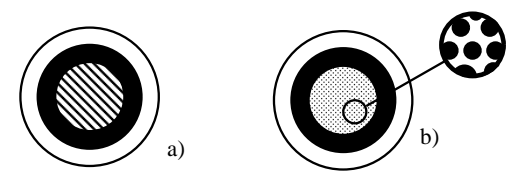

Figure 1: Sketch of the two electroding types of the front surface: the stripes are $200 \mu \mathrm{m}$ with an interdistance of the same width.

The emission results erratic as a function of samples and decaying with the shot number when the conventional grating front electrode, fig.1, is used. The aging effect, which leads to segregation of spontaneous polarization switching under the metal strips and to the screening of the domains of the uncovered zones, can be accounted for that behavior [4]. These segregation processes are avoided substituting the grating with an electrode consisting of a pattern of unconnected patches contained within a ring. The experimental results and a possible interpretation are presented.
The application of a $\geq 10 \mathrm{kV} / \mathrm{cm}$ electric field through the samples induces not only spontaneous polarization switching within the crystal, but also plasma formation on the cathode surface [2]. Both processes generate electrons, but the former generates a group of energetic electrons (FE electrons), whilst the latter generates only nonenergetic electrons.

\section{EXPERIMENTAL RESULTS}

Different patterns of metallic islands (fig.1) were tested: a) deposition of a uniform $100-\AA$ gold film (which autoarranges in separate patches); b) a very thin silver paste film, which auto-arranges as a patchwork; c) a uniform filling of the surface holes (the material is porous) with a carbon paste; and d) Au evaporation with a mask of $50-\mu \mathrm{m}-$ diam islands with an interdistance of $50 \mu \mathrm{m}$.

Samples without metal islands (with the external metal ring only) did not work; samples with a $50-\mu \mathrm{m}$ interdistance between islands worked badly. All the others always emitted, but the shorter the interdistance between the islands the more stable the emission.

The repetition rate was around $10 \mathrm{~Hz}$ for $8 / 65 / 35 \mathrm{sam}-$ ples, it was, instead, about $1 \mathrm{kHz}$ for 4/95/5 samples.

In the following, results obtained from hole filling and $8 / 65 / 35$ samples are reported. Only unprepoled ceramic at room temperature was used.

Only the energetic ferroelectric electrons are collected at the anode Faraday cup when the accelerating voltage is zero.

a) Negative pulse at the rear electrode

The signal obtained with $8 / 65 / 35$ material is reported in figure 2. First, the quite good stability is evident. The almost linear increase in the $8 / 65 / 35$ signal is explained by the continuous spontaneous polarization switching all along the excitation pulse.

The relaxation process of spontaneous polarization is longer than its buildup because the latter is driven by the fast voltage pulse, while the polarized state is metastable.

b) Positive pulse at the rear electrode

The emission signal is shown in fig. 3. Note the good stability of the signals with positive excitation. In this case, emission occurs at the end of the pulse, i.e., at relaxation of spontaneous polarization $P_{s}$.

In fig. 4 signals obtained with the negative HV pulse applied to the GE (front) electrode on 8/65/35 is reported.

In this experimental configuration the negative pulse sets an acceleration field for the whole emission phenomenon. 


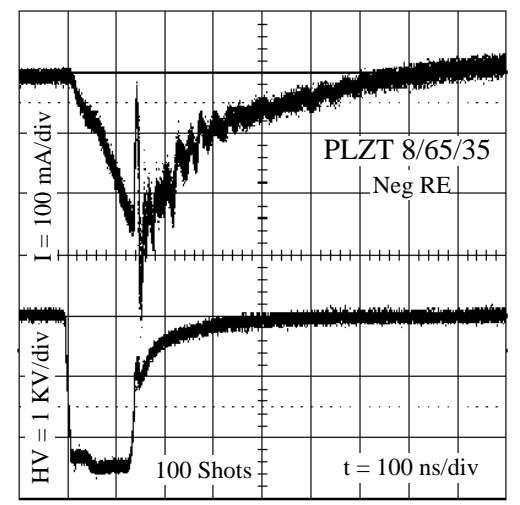

Figure 2: The superposition of 100 current signals, upper trace, and voltage signals, lower trace, is reported. The voltage pulse is applied to the rear electrode (RE), and the sample was a PLZT 8/65/35, as written in the frame, of 1 mm thickness.

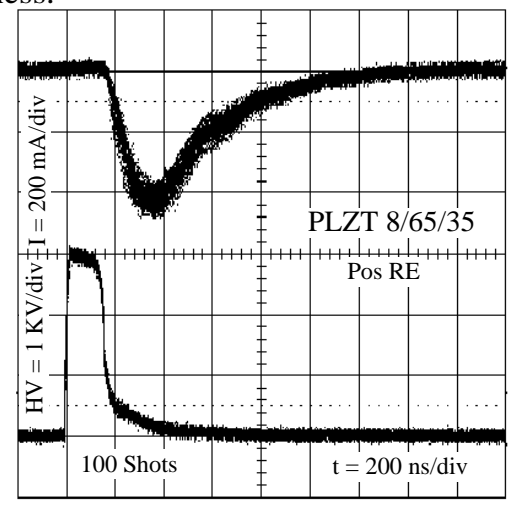

Figure 3: As in fig. 2 but with positive pulse to the rear electrode of a PLZT 4/95/5 sample.

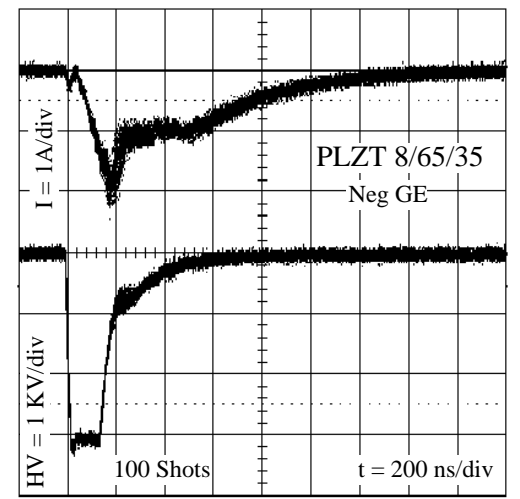

Figure 4: Superposition of 100 current signals, upper trace, and voltage signals, lower trace, are reported. The voltage pulse is applied to the front electrode (GE).

This explains the one order of magnitude higher amplitude of the current.

c) Negative pulse at the rear but with an accelerating field across the diode gap

The signals relative to $8 / 65 / 35$ samples are presented in figs. 5, and 6 . The scheme of the apparatus for these mea- surements is shown in 2

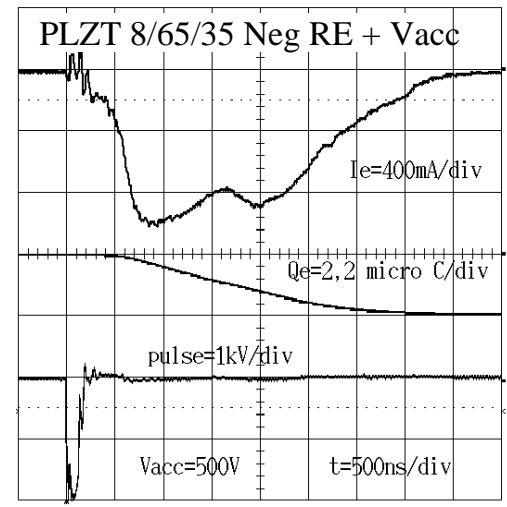

Figure 5: Signals of the current and of the charge whith an accelerating voltage of $500 \mathrm{~V}$.

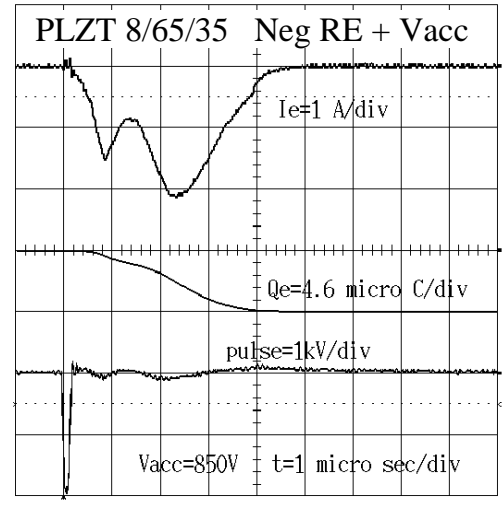

Figure 6: Ibidem as previous figure but with an accelerating voltage of $850 \mathrm{~V}$

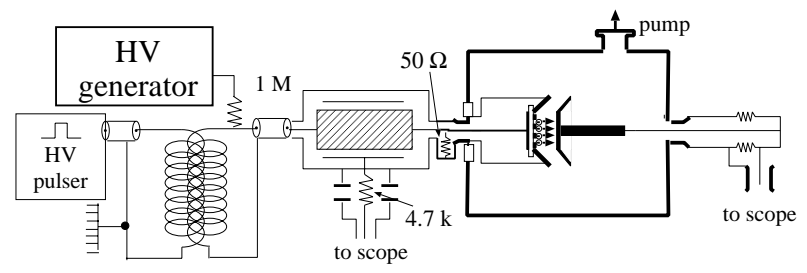

Figure 7: Sketch of the experimental apparatus used in tests with accelerating field

A probe tailored for the measurements of the high voltage pulse at the high voltage terminal has been developed.

The current signal has always two peaks: the first can be assigned to electrons emitted because of the ferroelectric switching, the second can be assigned to plasma produced electrons. The amplitude of the FE peak did not change doubling the accelerating voltage, in contrast with the plasma peak whose amplitude doubled. The emitted charge corresponds to the one stored in the buffer capacitors.

The signals were quite stable in shape and amplitude. 


\section{DISCUSSION}

The experimental observations obtained with samples electroded with grating could be lumped together in the concept of the crystal aging process. The neat separation of the cathode material in two parts, one under the metal strips and the other outside could induce a process of switching limitation within the covered zones and passivation of the uncovered surface.

The patchy electrode made the front surface homogeneous, preventing the segregation of the electric field and polarization switching. Our phenomenological view of electron emission is that the front of the voltage pulse propagates as a wave to the front surface, the induced polarization switching co-propagates with it, and spontaneous polarization sets through the whole sample; this polarization causes strong electric stress at the surface, which, in turn, changes the electric property of the surface from nonconductive to substantially conductive. This conclusion is supported by two facts: i) the measured switching current $\mathrm{dP} / \mathrm{dt}$ is the same as that obtained with samples having the surfaces completely metalized [3]; ii) in [6] a high mobility of the charge carriers within the stressed region was measured.

When the voltage excitation pulse is over, the charge carriers diffuse through the surface towards the external ring or through the bulk. The FE electron paths through the surface are not fixed because the surface state of a ferroelectric ceramic is dynamic and the surface is like a patchwork of pieces, whose properties [5] range from metallic to insulating. We recall that relaxors are in continuous evolution through metastable states, as in the spin-glass systems. Similar considerations also hold for antiferroelectric ceramics owing to the change of phase at each shot. It could also be possible that electrons traveling through the surface towards ground continuously rejuvanate the state of the surface.

The emission observed with an accelerating voltage tells that the switching activity has a time less than 1 microsecond. The amount of charge is of the order of $1 \mu \mathrm{C}$. The time of the plasma formation results around $1 \mu \mathrm{s}$. This matches with the time of its expansion over the surface (the diameter of the ring is about $6 \mathrm{~mm}$ ) with the estimated plasma velocity of $1 \mathrm{~cm} / \mu \mathrm{s}$.

\section{CONCLUSIONS}

Stable copious emission of energetic electrons from a ferroelectric ceramic disk, under the application of a fast highvoltage pulse, was obtained when the electrode of the emitting surface was a uniform pattern of unconnected metal patches contained within a metallic ring. This kind of front electrode avoids the aging process because the surface behaves like an almost homogeneous sheet, and the charge carriers migrate from the external ring to the dispersed points of the surface and viceversa through continuously changing paths. These two phenomena prevent any segregation either of the electric field or of polarization, and the material is continuously rejuvanated by the back and forth movements of the electrons through the surface and/or the bulk.

The plasma assisted emission can be exploited: the emitted charge is determined by the charge stored in the buffer capacitor.

\section{REFERENCES}

[1] H. Gundel, J. Handerek, H. Riege, E.J.N. Wilson and K. Zioutas, Ferroelectrics 100,1,1989.

[2] H. Riege, I. Boscolo, J. Handerek, U. Herleb, J. Appl. Phys. 84,1602, 1998.

[3] G. Benedek, I. Boscolo, J. Handerek, A. Moscatelli, A. Scurati, J. Appl. Phys, 83,2776,1998.

[4] I. Boscolo, A. Scurati, M. Stellato, 'The ferroelectric emission with suitable electroding of ceramic disks" J. Appl. Phys. in publ.

[5] K. Szot, W. Speier and W. Eberhardt, Appl. Phys. Lett. 60 (10), 1190-1192, 1992.

[6] W. Mock, Jr. and W. H. Holt J. Appl. Phys. 50, 2740, 1979. 